\title{
On a Unified Theory of Twocentre Harmonic Oscillator Integrals
}

\author{
I. The Onedimensional Oscillator \\ W. WitsCHEL \\ Physikalisch-Technische Bundesanstalt, Braunschweig \\ (Z. Naturforsch. 26 a, 940-942 [1971] ; received 3 March 1971)
}

\begin{abstract}
Twocentre harmonic oscillator overlap integrals, arbitrary transition integrals and collision energy etchange integrals for equal and different frequencies of the oscillators are contained in a generalized Franck-Condon-integral which is solved by operator methods in the second quantization representation.
\end{abstract}

\section{Introduction}

A generalized Franck-Condon-integral of the form

$$
I=\left\langle m, \omega, 0\left|x^{l}\right| r, \Omega, 0+b\right\rangle
$$

is important in the theory of electronic transitions in molecular spectroscopy ${ }^{1}$ and in the theory of the optical properties of colour centres ${ }^{2}$. For brevity a double bracket is introduced to indicate that the basis set for the frequencies $\omega$ and $\Omega$ is different. The integral reads now:

$$
\left.I=\left\langle m\left|x^{l}\right| r+b\right\rangle\right\rangle \text {. }
$$

The integral with $l=0$ is the usual twocentre overlap integral which was calculated by Hutchinson ${ }^{3}$. The theory of this integral was investigated with sophisticated analytical techniques by WAGNER ${ }^{4}$ and ANSBACHeR ${ }^{5}$. KOIDE ${ }^{6}$ introduced the occupation number representation in the calculation of the integral but he did not recognize that for different frequencies the basis systems of the oscillators are different.

Recently the interest in operator methods was renewed by $\operatorname{KATRIEL}^{7}$ who essentially gave an operator proof of Hutchison's calculations.

In Part 1 the present article aims at a new elementary solution of the generalized Franck-Condonintegral by applying a recently published operator method ${ }^{8}$. Part 2 discusses the generalized transition and kinetic energy integral and the collision energy exchange integral which are implicitely contained in the integral of Eq. (1).

1 G. Herzberg, Molecular Spectra and Molecular Structure, III. Electronic Spectra and Electronic Structure of Polyatomic Molecules, Van Nostrand Co., Princeton 1966.

2 H. Rampacher, Z. Naturforsch. 20 a, 350 [1965].

3 E. Hutchinson, Phys. Rev. 36, 410 [1930].

${ }_{4}$ M. WAGNER, Z. Naturforsch. 14 a, 81 [1959].

\section{Solution of the Integral I}

Following the notation of MessiaH ${ }^{9}$ and KA. TRIEL $^{7}$ the Hamiltonians of both oscillators are given by:

$$
\begin{aligned}
& \hat{H}=\hbar \omega\left(\hat{a}^{\dagger} \hat{a}+\frac{1}{2}\right), \\
& \hat{H}=\hbar \Omega\left(\hat{A}^{\dagger} \hat{A}+\frac{1}{2}\right) .
\end{aligned}
$$

The creation and annihilation operators are:

$$
\begin{aligned}
& \hat{a}=\left(\frac{M \omega}{2 \hbar}\right)^{1 / 2}\left(\hat{x}+\frac{i \hat{p}}{M \omega}\right), \\
& \hat{a}^{\dagger}=\left(\frac{M \omega}{2 \hbar}\right)^{1 / 2}\left(\hat{x}-\frac{i \hat{p}}{M \omega}\right), \\
& \hat{A}=\left(\frac{M \Omega}{2 \hbar}\right)^{1 / 2}\left(\hat{x}+\frac{i \hat{p}}{M \Omega}\right), \\
& \hat{A^{\dagger}}=\left(\frac{M \Omega}{2 \hbar}\right)^{1 / 2}\left(\hat{x}-\frac{i \hat{p}}{M \Omega}\right) .
\end{aligned}
$$

The commutators are:

$$
\left[\hat{a}, \hat{a}^{\dagger}\right]_{-}=\left[\hat{A}, \hat{A}^{\dagger}\right]_{-}=1 .
$$

Introducing abbreviations

$$
\begin{aligned}
& \varepsilon=\frac{1}{2}\left\{(\omega / \Omega)^{1 / 2}+(\Omega / \omega)^{1 / 2}\right\} \\
& \delta=\frac{1}{2}\left\{(\Omega / \omega)^{1 / 2}-(\omega / \Omega)^{1 / 2}\right\}
\end{aligned}
$$

yields

$$
\left.\begin{array}{l}
\hat{A}=\delta \hat{a}^{\dagger}+\varepsilon \hat{a} \\
\hat{A}^{\dagger}=\delta \hat{a}+\varepsilon \hat{a}^{\dagger}
\end{array}\right\}
$$

with the commutation relations

$$
\begin{aligned}
{[\hat{a}, \hat{A}]_{-} } & =\left[\hat{A^{\dagger}}, \hat{a}^{\dagger}\right]_{-}=\delta, \\
{\left[\hat{a}, \hat{A^{\dagger}}\right]_{-} } & =\left[\hat{A}, \hat{a}^{\dagger}\right]_{-}=\varepsilon .
\end{aligned}
$$

5 F. Ansbacher, Z. Naturforsch. 14 a, 889 [1959].

6 S. Koide, Z. Naturforsch. 15 a, 123 [1960].

7 J. Katriel, J. Phys. B, Atomic Molec. Phys. 3, 1315 [1970].

8 W. Witschel, J. Phys. B, Atomic Molec. Phys. 3, L 120 [1970].

9 A. Messiah, Quantum Mechanics, Vol. I, North Holland Publishing Company, Amsterdam 1964. 
The operators $\hat{A}^{\dagger}$ and $\hat{a}^{\dagger}$ generate the complete eigenvector system of the oscillators

$$
\begin{aligned}
& \left.|m\rangle\rangle=(m !)^{-1 / 2} \hat{A}^{\dagger m}|0\rangle\right\rangle, \\
& |m\rangle=(m !)^{-1 / 2} \hat{a}^{\dagger m}|0\rangle .
\end{aligned}
$$

In the derivation of the Franck-Condon-integral the same commutations will be used as in the derivation of the equal frequencies integral.

The integral to be solved is [see ${ }^{8}$, Eq. (4)]

$$
\left.I_{1}=\left\langle m\left|\hat{x}^{l} \exp \{i b \hat{p} / \hbar\}\right| r\right\rangle\right\rangle .
$$

The operator $\hat{p}$ is expressed by:

$$
\hat{p}=i\left(\frac{M \hbar \omega}{2}\right)^{3 / 2}\left(\hat{a}^{\dagger}-\hat{a}\right)
$$

which is introduced in Eq. (17). The result is:

$$
\left.I_{1}=\left\langle m\left|\hat{x}^{l} \exp \left\{-\alpha\left(\hat{a}^{\dagger}-\hat{a}\right)\right\}\right| r\right\rangle\right\rangle
$$

with

$$
\alpha=(M \omega / 2 \hbar)^{1 / 2} b .
$$

Applying Weyl's formula [see Appendix (A 3)] yields:

$I_{1}=\exp \left\{-\alpha^{2} / 2\right\}(m ! r !)^{-1 / 2}\left(\frac{\hbar}{2 M \omega}\right)^{l / 2}$

$$
\left.\left\langle 0\left|\hat{a}^{m}\left(\hat{a}+\hat{a}^{\dagger}\right)^{l} \exp \left\{-\alpha \hat{a}^{\dagger}\right\} \exp \{\alpha \hat{a}\} \hat{A}^{\dagger r}\right| 0\right\rangle\right\rangle .
$$

The essential point in the calculation of Eq. (21) is the commutation of $\exp \left\{-\alpha \hat{a}^{\dagger}\right\}$ to the left and $\exp \{\alpha \hat{a}\}$ to the right so that only the first terms of the series expansion will survive if they are working on the vacuum state. As $|0\rangle$ is different from $|0\rangle\rangle, \alpha \hat{a}$ is expressed by Eq. (12)

$$
\alpha \hat{a}=(\alpha / \varepsilon)\left(\hat{A}-\delta \hat{a}^{\dagger}\right) .
$$

The Weyl-formula is applied again:

$$
\begin{aligned}
I_{1}= & \exp \left\{-\alpha^{2} / 2\right\} \exp \left\{-\alpha^{2} \delta / 2 \varepsilon\right\} \\
& (m ! r !)^{-1 / 2}\left(\frac{\hbar}{2 M \omega}\right)^{l / 2}\langle 0| \hat{a}^{m}\left(\hat{a}+\hat{a}^{\dagger}\right)^{l} \\
& \left.\exp \left\{-\alpha\left(1+\frac{\delta}{\varepsilon}\right) \hat{a}^{\dagger}\right\} \exp \left(\frac{\alpha}{\varepsilon} \hat{A}\right) \hat{A}^{\dagger r}|0\rangle\right\rangle .
\end{aligned}
$$

The commutations can be easily performed by introducing the identity operator between $\langle 0|$ and $\hat{a}^{m}$ and between $|0\rangle\rangle$ and $\hat{A^{\dagger r}}$ [see Appendix (A 1)].

$$
\begin{aligned}
I_{1}=\exp \left\{-\frac{\alpha^{2}}{2}\left(1+\frac{\delta}{\varepsilon}\right)\right\}(m ! r !)^{-1 / 2}\left(\frac{\hbar}{2 M \omega}\right)^{l / 2} \\
\langle 0|\left\{\hat{a}-\alpha\left(1+\frac{\delta}{\varepsilon}\right)\right\}^{m} \\
\\
\left.\left\{\hat{a}^{\dagger}+\hat{a}-\alpha\left(1+\frac{\delta}{\varepsilon}\right)\right\}^{l}\left(\hat{A}^{\dagger}+\frac{\alpha}{\varepsilon}\right)^{r}|0\rangle\right\rangle .
\end{aligned}
$$

Now the operator $\hat{A}^{\dagger}$ is substituted by Eq. (12) :

$$
\begin{aligned}
I_{1}= & \exp \left\{-\frac{\alpha^{2}}{2}\left(1+\frac{\delta}{\varepsilon}\right)\right\}(m ! r !)^{-1 / 2}\left(\frac{\hbar}{2 M \omega}\right)^{l / 2} \\
& \langle 0|\left\{\hat{a}-\alpha\left(1+\frac{\delta}{\varepsilon}\right)\right\}^{m} \\
& \left.\left\{\hat{a}^{\dagger}+\hat{a}-\alpha\left(1+\frac{\delta}{\varepsilon}\right)\right\}^{l}\left(\delta \hat{a}+\varepsilon \hat{a}^{\dagger}+\frac{\alpha}{\varepsilon}\right)^{r}|0\rangle\right\rangle .
\end{aligned}
$$

As the operators $\hat{a}, \hat{a}^{\dagger}$ cannot work in the space of $|0\rangle\rangle$ the vacuum state of the second oscillator is expanded in terms of the eigenvectors of the first oscillator:

$$
\left.|0\rangle\rangle=\sum_{s} C_{0 s}|s\rangle, \quad C_{0 s}=\langle s \mid 0\rangle\right\rangle .
$$

The coefficients can easily be calculated by Hutchinson's method or by the following operator substitution in terms of $\langle 0 \mid 0\rangle\rangle$. This overlap integral was given by KATRIEL ${ }^{7}$ :

$$
\langle 0 \mid 0\rangle\rangle=\varepsilon^{-1 / 2} .
$$

Higher overlap integrals can be calculated using Eq. (12)

$$
\begin{aligned}
\left.C_{0 s}=(s !)^{-1 / 2}\left\langle 0\left|\hat{a}^{s}\right| 0\right\rangle\right\rangle \\
\left.=(s !)^{-1 / 2}(-1)^{s} \varepsilon^{-s}\left\langle 0\left|\left(\delta \hat{a}^{\dagger}-\hat{A}\right)^{s}\right| 0\right\rangle\right\rangle .
\end{aligned}
$$

The product can be calculated by simple multiplication. The matrix element vanishes if

$$
\left.\langle 0| \hat{a}^{\dagger} \text { or } \hat{A}|0\rangle\right\rangle \text {. }
$$

It can be seen that only even powers of $s$ give nonzero results. For example:

$$
\left.C_{01}=0, \quad C_{02}=-2^{-1 / 2}(\delta / \varepsilon)\langle 0 \mid 0\rangle\right\rangle .
$$

The final result is:

$$
\begin{gathered}
I_{1}=\exp \left\{-\frac{a^{2}}{2}\left(1+\frac{\delta}{\varepsilon}\right)\right\}(m ! r !)^{-1 / 2}\left(\frac{\hbar}{2 M \omega}\right)^{l / 2} \\
\cdot \sum_{s} C_{0 s}(s !)^{-1 / 2}\langle 0|\left\{\hat{a}-\alpha\left(1+\frac{\delta}{\varepsilon}\right)\right\}^{m} \\
\left.\cdot\left\{\hat{a}+\hat{a}^{\dagger}-a\left(1+\frac{\delta}{\varepsilon}\right)\right\}^{l}\left(\delta \hat{a}+\varepsilon \hat{a}^{\dagger}+\frac{\alpha}{\varepsilon}\right)^{r} \hat{a}^{\dagger s}|0\rangle\right\} .
\end{gathered}
$$

As there is only a limited number of annihilation operators available the series breaks off after few terms. The matrix elements can be calculated by elementary algebra:

$\left\langle 0\left|\hat{a}^{m} \hat{a}^{\dagger m}\right| 0\right\rangle=m$ ! [see Appendix (A 2)].

Eq. (31) contains the result for the equal frequencies Franck-Condon-integral ${ }^{8}$ as a special case:

$$
\begin{aligned}
I_{2}= & \exp \left\{-\alpha^{2} / 2\right\}(m ! r !)^{-1 / 2}\left(\frac{\hbar}{2 M \omega}\right)^{l / 2} \\
& \cdot\left\langle 0\left|(\hat{a}-\alpha)^{m}\left(\hat{a}+\hat{a}^{\dagger}-\alpha\right)^{l}\left(\hat{a}^{\dagger}+\alpha\right)^{r}\right| 0\right\rangle .
\end{aligned}
$$




\section{Integrals Related to the Generalized Franck-Condon-Integral}

\subsection{Transition Integral}

If $\alpha$ equals zero the integral is reduced to a transition integral between oscillators of different frequencies but equal equilibrium distances.

$\left.I_{3}=\left\langle m\left|\hat{x}^{l}\right| r\right\rangle\right\rangle=(m ! r !)^{-1 / 2}(\hbar / 2 M \omega)^{l / 2}$

$\cdot\left\{\sum_{s} C_{0 s}(s !)^{-1 / 2}\left\langle 0\left|\hat{a}^{m}\left(\hat{a}+\hat{a}^{\dagger}\right)^{l}\left(\delta \hat{a}+\varepsilon \hat{a}^{\dagger}\right)^{r} \hat{a}^{\dagger s}\right| 0\right\rangle\right\}$.

For equal frequencies the result is:

$$
\begin{aligned}
I_{4}=\left\langle m\left|\hat{x}^{l}\right| r\right\rangle= & (m ! r !)^{-1 / 2}(\hbar / 2 M \omega)^{l / 2} \\
& \cdot\left\langle 0\left|\hat{a}^{m}\left(\hat{a}+\hat{a}^{\dagger}\right)^{l} \hat{a}^{\dagger r}\right| 0\right\rangle .
\end{aligned}
$$

\subsection{Kinetic Energy Integral}

In some spectroscopic applications the kinetic energy integral is important. This integral can be generalized for arbitrary powers of the momentum operator.

$$
\left.I_{5}=\left\langle m\left|\hat{p}^{l}\right| r+b\right\rangle\right\rangle .
$$

By a slight change of notation in Eq. (31) the result is:

$$
\begin{gathered}
I_{5}=\exp \left\{-\frac{a^{2}}{2}\left(1+\frac{\delta}{\varepsilon}\right)\right\}(m ! r !)^{-1 / 2} i^{l}\left(\frac{M \hbar \omega}{2}\right)^{l / 2} \\
\cdot\left\{\sum_{s} C_{0 s}(s !)^{-1 / 2}\langle 0|\left\{\hat{a}-\alpha\left(1+\frac{\delta}{\varepsilon}\right)\right\}^{m}\right. \\
\left.\left\{\hat{a}^{\dagger}-\hat{a}+\alpha\left(1+\frac{\delta}{\varepsilon}\right)\right\}^{l}\left(\delta \hat{a}+\varepsilon \hat{a}^{\dagger}+\frac{\alpha}{\varepsilon}\right)^{r} \hat{a}^{\dagger s}|0\rangle\right\} .
\end{gathered}
$$

For equal frequencies this result simplifies to:

$I_{6}=\left\langle m\left|\hat{p}^{l}\right| r+b\right\rangle=\exp \left\{-\alpha^{2} / 2\right\}(m ! r !)^{-1 / 2} i^{l}$

$\left.\cdot(M \hbar \omega / 2)^{l / 2}\langle 0| \hat{a}-\alpha\right)^{m}\left(\hat{a}^{\dagger}-\hat{a}+\alpha\right)^{l}\left(\hat{a}^{\dagger}+\alpha\right)^{r}|0\rangle$.

\subsection{Collisionenergy-exchange Integral}

A convenient interaction in collision processes leads to interaction integrals of the form

$$
\left.\left(\frac{\hbar}{2 M \omega}\right)^{l / 2}\left\langle m\left|\left(\hat{a}^{\dagger}+\hat{a}\right)^{l} \exp \gamma\left(\hat{a}^{\dagger}+\hat{a}\right)\right| r\right\rangle\right\rangle
$$

( $\gamma$ is an arbitrary constant)

$\left(\right.$ TAKAYANAGI $\left.{ }^{10}\right)$. Similar integrals are important if an interatomic potential like the Morse-potential is calculated in an harmonic oscillator basis. Again the results can be writen down using Eq. (31) after a slight change of notation:

10 K. Takayanagi, Advances Atomic Molecular Physics 1, 149 [1965].

$$
\begin{aligned}
I_{7}= & \exp \left\{\frac{\gamma^{2}}{2}\left(1+\frac{\delta}{\varepsilon}\right)\right\}(m ! r !)^{-1 / 2}\left(\frac{\hbar}{2 M \omega}\right)^{l / 2} \\
& \left\{\sum_{s} C_{0 s}(s !)^{-1 / 2}\langle 0|\left\{\hat{a}+\gamma\left(1+\frac{\delta}{\varepsilon}\right)\right\}^{m}\right. \\
& \left.\left\{\hat{a}+\hat{a}^{\dagger}+\gamma\left(1+\frac{\delta}{\varepsilon}\right)\right\}^{l}\left(\delta \hat{a}+\varepsilon \hat{a}^{\dagger}+\frac{\gamma}{\varepsilon}\right)^{r} \hat{a}^{\dagger s}|0\rangle\right\} .
\end{aligned}
$$

For equal frequencies Eq. (40) simplifies to

$$
\begin{aligned}
I_{8}= & \exp \left\{\gamma^{2} / 2\right\}(m ! r !)^{-1 / 2}(\hbar / 2 M \omega)^{l / 2} \\
& \cdot\left\langle 0\left|(\hat{a}+\gamma)^{m}\left(\hat{a}+\hat{a}^{\dagger}+\gamma\right)^{l}\right|\left(\hat{a}^{\dagger}+\gamma\right)^{r} \mid 0\right\rangle .
\end{aligned}
$$

\section{Conclusion}

Second quantization and operator technique leads to elementary expressions for some important harmonic oscillator integrals which simplify the calculation of transition probabilities between arbitrary oscillator states considerably. It is difficult to treat $n$-dimensional oscillators in a similar manner because additional angular momentum quantum numbers are important. A simple expression can be given if only integrals of the type

$$
\left.I=\left\langle m\left|x_{k}^{l}\right| r+b\right\rangle\right\rangle
$$

are considered; $x_{k}$ is the normal coordinate of mode $k$ and the state vectors $|m\rangle$ and $|r\rangle$ are of the form:

$$
\begin{aligned}
|m\rangle & =\left|m_{1} \ldots m_{k} \ldots\right\rangle, \\
|r\rangle\rangle & \left.=\left|r_{1} \ldots r_{k} \ldots\right\rangle\right\rangle .
\end{aligned}
$$

In this case the generalized Franck-Condon-integrals for $n$-dimensional oscillators are products of the linear harmonic oscillator integral Eq. (31).

Discussion with Professors Reich and Richter are gratefully acknowledged.

\section{Appendix}

\section{Collection of Some Operator Formulas} Used in the Text

$\exp \{\beta \hat{B}\} \hat{C} \exp \{-\beta \hat{B}\}=\hat{C}+\beta[\hat{B}, \hat{C}]$

$$
\begin{aligned}
& +\frac{\beta^{2}}{2}\left[\hat{B},[\hat{B}, \hat{C}]_{-}\right]_{-}+\ldots=\sum_{n=0}^{\infty} \frac{\beta^{n}}{n !}\{\hat{B}, \hat{C}\}_{-} \\
& \exp \{\beta \hat{B}\} \hat{C}^{m} \exp \{-\beta \hat{B}\} \\
& =(\exp \{\beta \hat{B}\} \hat{C} \exp \{-\beta \hat{B}\})^{m}, \quad \text { (A 1) } \\
& \hat{a}^{m} \hat{a}^{\dagger m}=\prod_{p=1}^{m}\left(\hat{a}^{\dagger} \hat{a}+p\right), \\
& \hat{a}^{\dagger m} \hat{a}^{m}=\prod_{p=1}^{m}\left(\hat{a}^{\dagger} \hat{a}+1-p\right),
\end{aligned}
$$$$
\exp \{\hat{B}+\hat{C})=\exp \hat{B} \exp \hat{C} \exp \left(-\frac{1}{2}[\hat{B}, \hat{C}]_{-}\right)
$$

if: $\left[\hat{B},[\hat{B}, \hat{C}]_{-}\right]_{-}=\left[\hat{C},[\hat{B}, \hat{C}]_{-}\right]_{-}=0$.

This formula is named occasionally Weyl-, Zassenhaus-, Baker-Hausdorff-Campbell- or, after a clear proof ${ }^{9}$, Glauber-formula. 